\title{
Theoretical and experimental applications of the flying spot camera
}

\section{by C.GRUSS and D.BALAGEAS}

\author{
ONEAA, Thermophysics Division, BP72, 92322 CHATILLON, France.
}

\begin{abstract}
Tho principle of the flying spot camera, a non destructive testing system, is to heat a sample with a moving laser spot and to observe the time evolution of the temperature with an IR detector viewing an aren attached to the laser spot with a constant offset. In this paper, to obtain a better understanding of the axperimental data, models are developed for several cases : semi-infinite adiabatic solid, solid of finite Hhlcknoss, infinite vertical crack, effect of convective losses, effect of an optical penetration of the laser boam. For all theses cases analytical solutions are proposed. Two experimental set-up are described: thelr performances are discussed and some experimental results are compared with the theory.
\end{abstract}

\section{Nomenclature}

$G \rho$ hoat capacity per unit volume

d detectorspot size

sample thickness $V$ laser velocity

$R \quad$ laser beam radius
$P_{O}$ laser power
$V \quad$ laser velocity
$x, y, z$ coordinates

Gimonsionless parameters:

$$
\begin{aligned}
& X^{*}=x / A \\
& V^{*} \quad R V / \kappa \\
& b / K R / k \text { Biot number }
\end{aligned}
$$

\section{Introduction}

The aim of this paper is to present some theoretical studies and experimental data of the photothermal camera also called flying-spot camera. Its principle is to heat a sample with a gcanning $\mathrm{CW}$ laser and to detect the surface infrared emission of a point following the laser spot at a given and adjustable offset. This type of camera has been developed in the U.S.A for years by E..J.KUBIAK [1] and more recently by R.L.THOMAS'S team from Wayne State University [2]. Guch a system provides two main improvements with respect to the conventional stimulated infrared thermography using uniform illumination : a very short response time making possible the study of thin coatings, and a good sensitivity to interfaces perpendicular to the surface. The first puirt of this paper presents some theoretical studies of the flying spot camera applications. The calculations are based on the use of the Green's functions. This method leads to analytical golutlons for a rather large number of cases : coating, infinite vertical crack, semi-infinite solid with convective heat losses. The second part presents the experimental set-up. In the third part gome measurements are presented.

\section{Theory}

\subsection{Introduction}

Lot us consider an isotropic and homogeneous sample with constant thermal characteristics and plane boundaries. The heat transfer equation to solve is :

$$
k \Delta T+P(x, y, z, t)=C \rho \frac{\partial T}{\partial t}
$$

The initial and boundary conditions vary with the problem to solve, but we always consider that there are no heat losses on the sample's surface (except for the convection problem). To wolve equation (1) we use the Green functions (see for example [3] p.353) which consists in laking a Dirac function as power density. The final solution is given by convolution of the Green 
function with the real power density distribution. The Green function that we consider is well known; we give its application to the moving laser heating.

\subsection{The semi-Infinite solid}

Let us consider the half space $z \geq 0$ with no heat losses on the surface and the initial temperature taken as the reference. The power distribution of the laser source takes the following form:

$$
P(x, y, z, t)=2 \alpha P_{o} \exp \left\{-2\left[(x-V t)^{2}+y^{2}\right] / R^{2}\right\} 2 \delta(z) / \pi R^{2} \text { for } z>0
$$

where $P O$ is the power of the laser beam, $R$ its radius, $V$ its velocity, $\alpha$ the sample absorptivity (we assume a zero absorption depth), $\delta(z)$ the Dirac function. This calculation has been made in [4]. The laser spot moving along the $x$-axis, the solution for all values of time is derived from the solution for $t=0$, using the formula :

$$
T(x, y, t)=T(x-v t, y, 0)
$$

The solution at $t=0, z=0$ (we only are interested by the surface temperature) is:

$$
T=\frac{\alpha P o}{\pi k R} \sqrt{\frac{2}{\pi}} T^{*}\left(X^{*}, Y^{*}, V^{*}\right)
$$

with the dimensionless temperature:

$$
T^{*}\left(X^{*}, Y^{*}, V^{*}\right)=\int_{0}^{+\infty} \exp \left(-2 l\left(X^{*}+V^{*} F_{O}\right)^{2}+Y^{*} 2 /\left(1+8 F_{O}\right)\right\} \frac{d \sqrt{8 F_{0}}}{1+8 F_{0}}
$$

Figure 1 shows the evolution of normalized temperature (ratio of the temperature $T^{*}$ to the maximum temperature $T^{*} \max$ for a given velocity $\left.V^{*}\right)$ vs $X^{*}$ for different velocities. The higher the spot velocity, the lower the $x$-gradient of the temperature ; when $V^{*}$ tend to infinity then $T^{*}$ becomes proportionnal to $1 / \sqrt{ } X^{*}$. This means that higher is the spot velocity, closer is the phenomenon to the 1-D cooling.

\subsection{The slab}

We consider a slab $(0 \leq z \leq e)$, with no heat losses at $z=0$ and , for $z=e$, the following possibilities : no heat losses (case a) or zero prescribed temperature (case b) ; these two cases are the limits of a two-layer material, with the second layer made of respectively a perfect insulator and a perfect conductor. Both related Green's functions are well kown and the calculation \{with the power density (2)\}leads to the following dimensionless temperature :

$$
T^{*}\left(X^{*}, Y^{*}, V^{*}\right)=\int_{0}^{+\infty} H \exp \left\{-2\left(X^{*}+V^{*} F o\right)^{2} /\left(1+8 F_{0}\right)\right\} \frac{d \sqrt{8 F o}}{1+8 F_{0}}
$$

with: $\quad H=1+2 \sum_{n=1}^{n=\infty}(-1)^{m} \exp \left(-n^{2} / F_{0}\right)$

$m=0$ (case a) or $m=n$ (case b) .

The Fourier number related to the thickness is $\mathrm{Fo}_{e}=k_{\mathrm{t}} \mathrm{e}$. Figure 2 illustrates the temperature profile dependence on the thickness vs $X^{*}$. The upper curve is related to the insulated slab, the median curve to the semi-infinite solid, the lower curve to the slab with prescribed temperature. The higher the spot velocity or the thickness, the closer the three curves. This mean that the depth of detectable defect decreases when the spot velocity increases. Note that the case a is equivalent to an infinite thermal resistance of depth $e$ and parallel to the surface.

\subsection{The vertical crack}

Let us consider the semi-infinite medium with no heat losses at the surface $z=0$ and an infinite plane thermal resistance at $X=X_{r}$ (no heat flow across the plane $\left.X=X_{r}\right)$; this is the simplest model for a vertical thermal resistance. A power density (2), gives at $t=0$ :

- for $x>X_{r}: \quad T^{*+}\left(X^{*}\right)=T^{\star} c\left(X^{*}\right)+T^{\star} C\left(2 X_{r}^{*}-X^{*}\right)$

- for $x<X_{r}: \quad T^{*-}\left(X^{*}\right)=T^{*} O\left(X^{*}\right)+T^{*} O\left(2 X_{r^{*}}-X^{*}\right)-T^{*+}\left(X^{*}\right)$ 
with $X_{r}^{*}=X_{r} / R, T^{\star O}$ given by $(5)$ and $T^{*}$ by $(6)$ with :

$$
H=0.5+0.5 e r f\left\{\left[X_{r}^{*}-X^{*}+8 F o\left(X_{r}^{*}+F_{o V^{*}}\right)\right] \sqrt{\left.4 F o\left(1+8 F_{o}\right)\right\}}\right.
$$

The previous figures present space distribution of the temperature ; this space distribution is (1) same as a time distribution; but it isn't true in the case of a vertical crack, because the relation (3) romains not verified. Figure 3 presents a flying spot signal for different offsets $\left(\Delta X^{*}=\Delta X / R\right)$ botween the laser and the detector spot (the offset is positive when the detector spot follows the lasor spot). The laser pass througth the vertical crack at $F O=0$; so the detector spot pass Ilirougth the crack at $F O=\Delta X^{*} / N^{*}$. This figure shows that to have the best detection you should thko $\Delta X^{*}$ between 0 and -0.5 .

\subsection{The semi-Infinite solid with convective losses}

Lot us consider the semi-infinite solid with the following boundary condition:

$$
k \frac{\pi}{\partial z}=h T
$$

If 19 the convective heat transfer coefficient. The Green function can be found by the Laplace Iransformation leading for the dimensionless temperature to an expression like (6) with:

$$
H=1-B \sqrt{\pi F O} \exp \left(B i^{2} \text { Fo)erfd }(B i \sqrt{F O})\right.
$$

with the Biot number $B i=h R / k$ relative to the laser beam radius as the characteristical parameter of the convective losses. Figure 4 shows the influence of convective losses on the temperature space (or time) distribution. The higher the velocity, the lower the sensivity to the convective logses.

\section{6. The seml-InfInite medium with a volume absorption}

To take into account the optical penetration of the laser beam into the sample, a power distribution $\exp \left(-z / \lambda_{0}\right) / \lambda_{0}$ ( $\lambda_{0}$ absorption depth) has to be taken instead of $2 \delta(z)$ in (2). For the $6 \mathrm{em} /$ infinite medium, the dimensionless temperature is given by formula (6) with :

$$
H=\sqrt{\pi} Z \exp \left(Z^{2}\right) \operatorname{erfc}(Z)
$$

with $Z \sqrt{k t} / \lambda_{0}$. The dimensionless absorption depth $\lambda_{0}{ }^{*}=\lambda_{0} / R$ is equivalent to the inverse of Ulie Blot number for the convection problem. Figure 5 presents the space (or time) temperature distribution for several $\lambda 0^{*}$, for $V^{*}=100$. The effects are very important and cannot be neglected when $\lambda_{0}$ is greater than 0.1 of the laser spot. The higher the velocity, the higher the effect of the optloal penetration.

\subsection{The effect of space integration - other cases}

Tho If detection is made on a small area and not on a single point. This space convolution can ditgtort the measured temperature distribution and can be studied in the geometrical optics approximation. For the semi-infinite adiabatic solid and for a square spot detector of size $d$, the integration effect is negligible when $d$ is smaller than $R / 2$.

$T$ The Green function can be determined for several other cases : the wedge with prescribed tomperature or with no heat losses at its surface; the two-layer adiabatic semi-infinite solid with peifleol Interface ; the semi-infinite solid with an horizontal or vertical plane thermal resistance of Inilto value. All these examples show that analytical solutions for complex problems can be botainod with the Green function method.

\section{Exporimental set-up}

Tho lirst experimental set-up (static detection) used is shown figure 6 . The set-up consists in an argon laser whose beam is reflected on a scanning mirror toward the sample, and in an infrared HgCdTo detector which is focalized by a $20 \mathrm{~cm}$ focal length lens on the sample. The fixed spot of thie If detector is on the laser spot trajectory. The distance between the detector lens and the ammple is $50 \mathrm{~cm}$; the diameter of the lens is $5 \mathrm{~cm}$. This leads to a theoretical temperature regolullon of $0.2 \mathrm{~K}$. The measured temperature resolution is $0.4 \mathrm{~K}$ for a bandwidth limit of $1.3 \mathrm{kHz}$. The laser beam velocity can vary from a few $\mathrm{cm}_{\mathrm{s}} \mathrm{s}^{-1}$ to a few $\mathrm{m} . \mathrm{s}^{-1}$. The advantage of this set-up 1610 allow the observation of the entire heating and cooling of the sample due to the laser; and the second set-up only observes the space variations of one given point of the temperature time 10 sponso. 


\section{http://dx.doi.org/10.21611/qirt.1992.004}

Figure 7 shows a classical flying spot set-up in which the IR detector spot follows the laser spot : this is obtained by coupling the laser beam with the IR detector by a flat elliptic mirror which deviates the laser beam from $90^{\circ}$. The laser beam and the detector spot are sent together towards the sample thanks to the scanning system. The aperture of the scanning system is 5 $\mathrm{cm}$, the sample is $50 \mathrm{~cm}$ from it, and the detector lens is $25 \mathrm{~cm}$ farther from sample than the scanning system. The theoretical temperature resolution in these conditions is only $0.5 \mathrm{~K}$ and $1 \mathrm{~K}$ measured resolution.

\section{Results}

Figures 8 to 11 present some results obtained with the static detection set-up and figure 12 and 13 results obtained with the flying spot set-up.

Figure 8 shows the normalized temperature profile for a sample of black plexiglas with two laser velocities $\left(10\right.$ and $\left.40 \mathrm{cms}^{-1}\right)$. The experimental parameters are : $k=0.2 \mathrm{~W} \cdot \mathrm{m}^{-1} 1 \mathrm{~K}, \mathrm{k}=0,1 \mathrm{~mm}^{2} \mathrm{~s}^{-1}$, $\varepsilon=0,95$ (experimentaly measured); with an absorption depth of $\lambda o^{*}=3 / 4$, a good agreement between theory and experiment is obtained. Direct transmission measurement gives an absortion depth for the plexiglas of $0.2 \mathrm{~mm}$, in agreement with the previously reported measurement. The maximum temperature in the experimental conditions would be $500^{\circ} \mathrm{C}$ for $\lambda_{0}=$ 0 instead of $50^{\circ} \mathrm{C}$ for $\lambda_{0}=3 R / 4$.

Figure 9 shows the effect of convective losses produced by a fan on a plexiglas sample; the laser beam velocity is $1 \mathrm{~cm} . \mathrm{s}^{-1}$. Curve fitting identification leads to the value $h=200 \mathrm{~W} . \mathrm{m} .-2 \mathrm{~K}-1$ for the heat transfer coefficient.

Figure 10 presents the effect of a black paint on the response of a pasteboard specimen : the normalized temperature distribution is given versus time. The laser spot velocity is $22.4 \mathrm{~cm} . \mathrm{s}^{-1}$. The upper curve is related to the pasteboard without paint: the curve fitting identification gives an absorption depth of $0.1 R=30 \mu \mathrm{m}$. The black paint has a good absorbtion: some optical measurement give an absorption depth $\lambda_{0} \leq 5 \mu \mathrm{m}$. The painted specimen experiences a cooling which is faster than without paint. The experiment demonstrates that the paint effect shoudn't be neglected. Nevertheless, a quantitative analysis of this measurement is difficult since the variability of the paint characteristics are unkwown (emissivity, diffusivity, thickness).

Figure 11 shows the effect of thickness on a sample of (black paint) copper: the first measurement was made with $0.5 \mathrm{~cm}$ thickness and the second with $0.1 \mathrm{~mm}$. We can consider the first sample as a semi-infinite adiabatic solid (lower curve) and the second one as an adiabatic slab (upper curve). The laser beam velocity is $V=4 \mathrm{~cm} / \mathrm{s}$.

Figure 12 presents a flying spot image compared with the equivalent IR camera (obtained by using the flying spot set-up without laser). The sample is a pasteboard of $5 \mathrm{~cm}$ length with a $2 \mathrm{~cm}$ ribbon of copper stuck in the middle of it ; some black paint covers the sample. The laser velocity is $9.5 \mathrm{~cm} / \mathrm{s}$. The IR camera measurement (lower curve) only sees the black paint; but the flying spot camera (upper curve) detects the pasteboard and the copper under the paint. This experiment illustrate one interest of the flying spot camera.

\section{Conclusion}

The theory gives good results when it takes account of different experimental effects : effect of optical penetration, detector space integration. It can be developed to take account of the non infinite value of the thermal resistance, the non infinite size of a resistance. The static detection set-up gives results which can easily be compared with the theory and helps us for the choice of technical solution for the flying spot set-up. These last set-up must be developed to give images and not only an isolated line. This will be soon done with some improvement of the temperature resolution.

\section{REFERENCES}

[1] KUBIAK (E.J.). - Infrared defection of fatigue cracks and oher near-surface defects - Applied Optics, September 1968 , Vol .7, $N^{\circ} 9$, p.1743.

[2] WANG (Y.Q.), KUO (P.K.), FAVRO (L.D.) and THOMAS (R.L.). - A novel "flying-spot" infrared camera for imaging very fast thermal phenomena. - Springer Series in Optical Science, Vol 62, Photoacoustic and Photothermal Phenomena II ,1990.

[3] CARSLAW (H.S.) and JAEGER (J.C.). - Conduction of heat in solids. - Oxford University Press, 1959.

[4] CLINE (H.E.) and ANTHONY (T.R.). - Heat treating and welting material with a scanning laser or

electron-beam. -- Journal of Applied Physics, Vol 48, September 1977. 


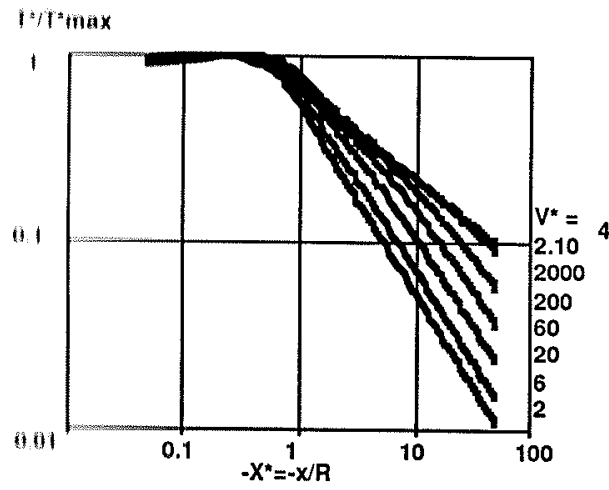

1ig: 1. - Normalised temperature profile for the semi-infinite solid

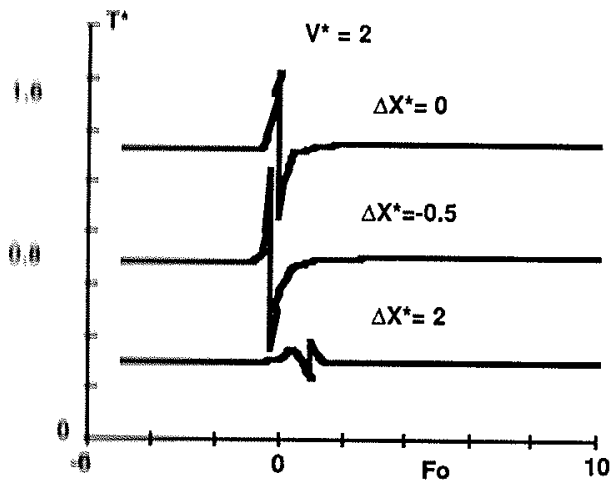

19. 3. Temperature profile for the vertical crack - flying spot line

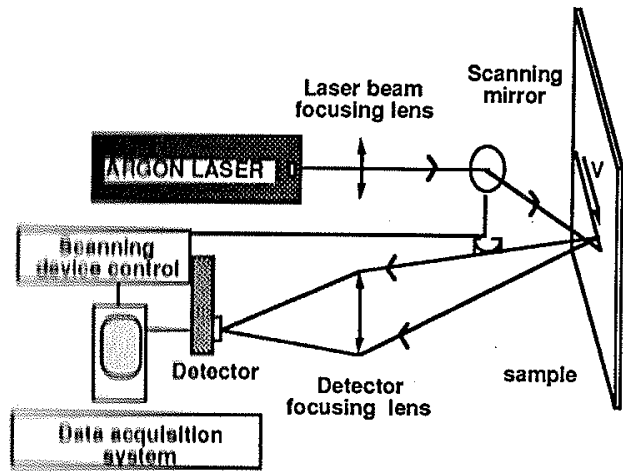

FIg. 6. - Statlc detection set-up

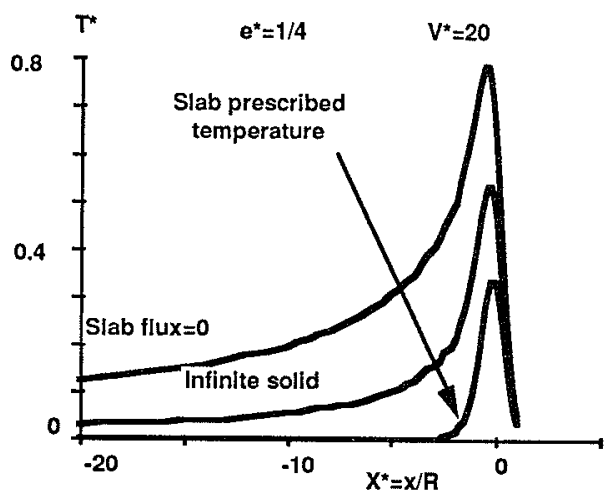

Fig. 2. - Effect of thickness on the temperature profile

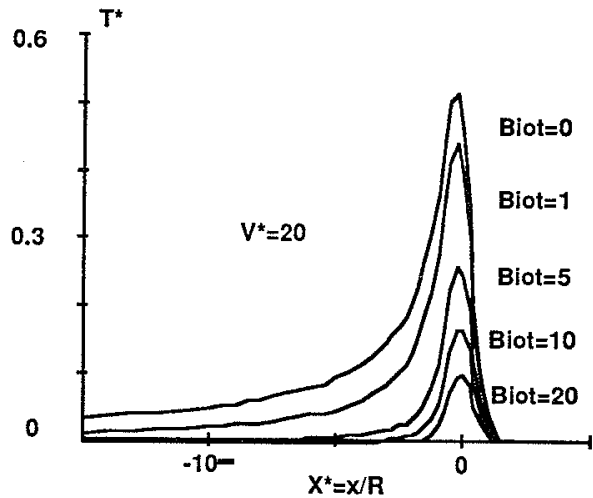

Fig. 4. - Effect of convective losses

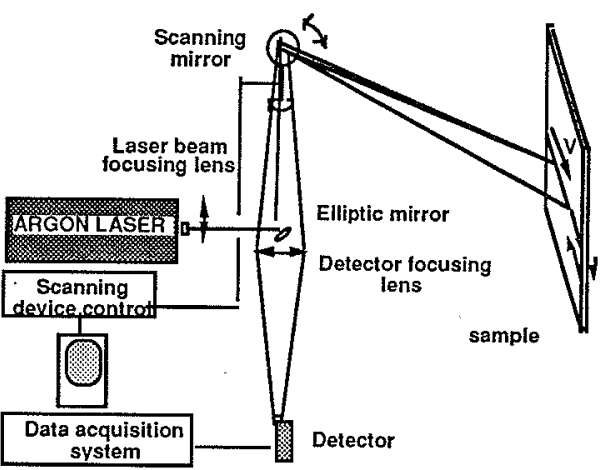

Fig. 7. - Classical flying spot set-up 
http://dx.doi.org/10.21611/qirt.1992.004

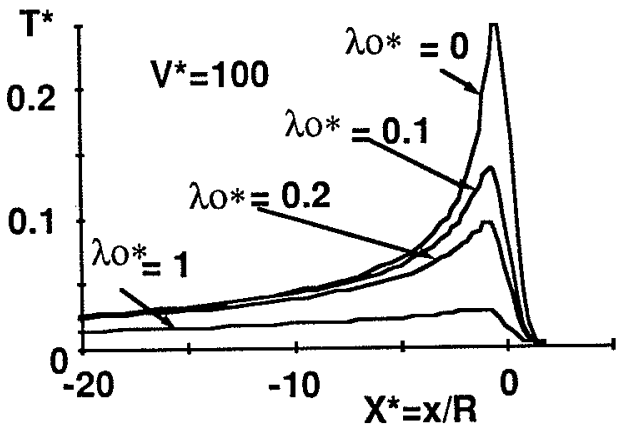

Fig. 5. - Effect of optical penetration

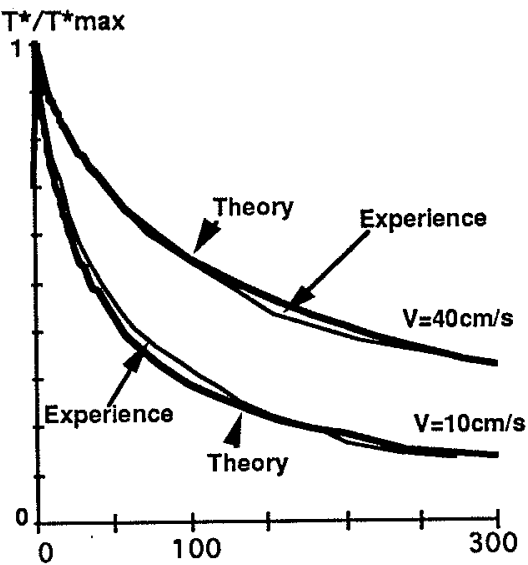

Fig 8: Experience with a plexiglas sample

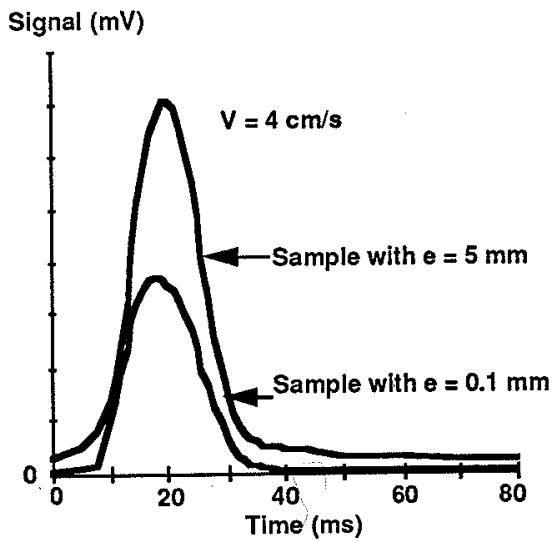

Fig 11: Effect of thickness on a paint

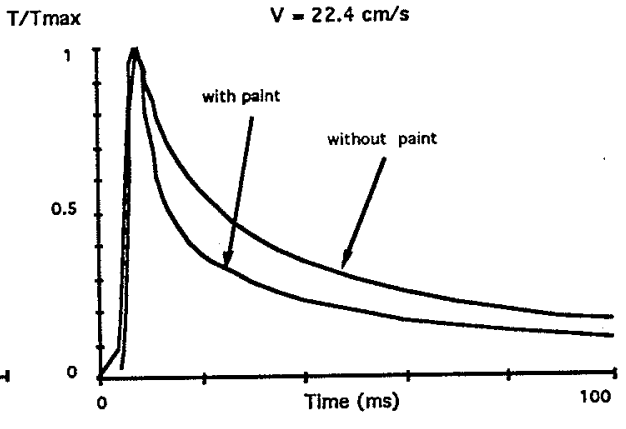

Fig. 10. - Effect of paint on a pasboard sample

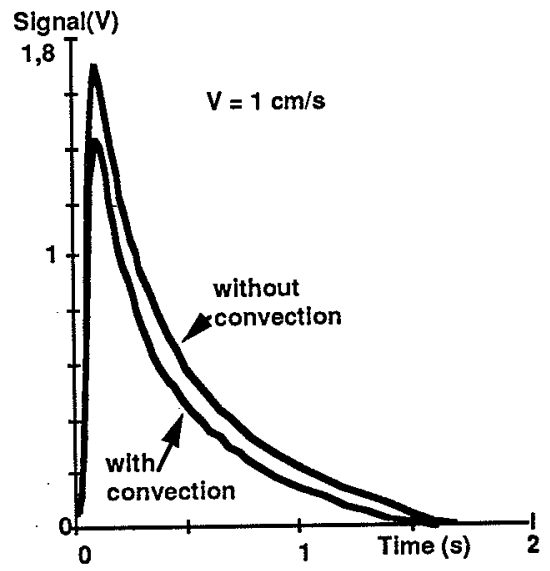

Fig 9 : Experience with plexiglas sample: effect of convection

Signal (V)

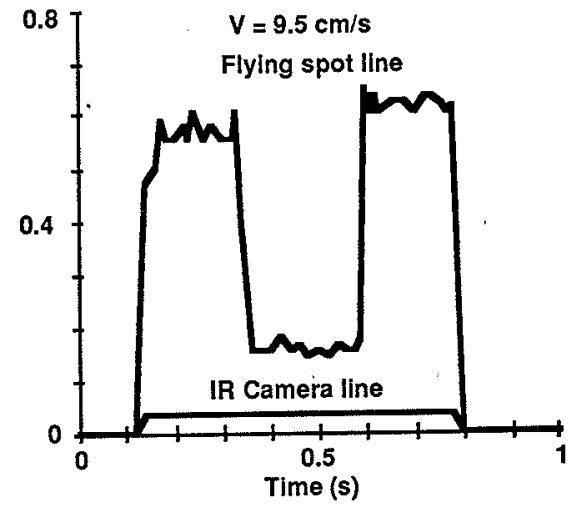

Fig 12 : Flying spot Image of a sample with different material under paint coat 